\title{
Physically Secure Wearable-Wearable Through-Body Interhuman Body Communication
}

\author{
David Yang *, Shovan Maity and Shreyas Sen \\ SPARC Lab, Department of Electrical and Computer Engineering, Purdue University, West Lafayette, IN, United States
}

Human body communication ( $\mathrm{HBC}$ ) has recently emerged as an alternative method to connect devices on and around the human body utilizing the electrical conductivity

OPEN ACCESS

Edited by:

YuWu,

University College London,

United Kingdom

Reviewed by:

Jeremy Gummeson,

University of Massachusetts Amherst,

United States

Wala Saadeh,

Lahore University of Management

Sciences, Pakistan

Chenyu Tang,

Beihang University, China

*Correspondence:

David Yang

yang996@purdue.edu

Specialty section:

This article was submitted to

Wearable Electronics,

a section of the journal

Frontiers in Electronics

Received: 01 November 2021 Accepted: 31 December 2021

Published: 03 February 2022

Citation:

Yang D, Maity S and Sen S (2022)

Physically Secure Wearable-Wearable

Through-Body Interhuman

Body Communication.

Front. Electron. 2:807051.

doi: 10.3389/felec.2021.807051 properties of the human body. HBC can be utilized to enable new interaction modalities between computing devices by enhancing the natural interaction of touch. It also provides the inherent benefit of security and energy-efficiency compared to a traditional wireless communication, such as Bluetooth, making it an attractive alternative. However, most state-of-the-art HBC demonstrations show communication between a wearable and an Earth ground-connected device, and there have been very few implementations of HBC systems demonstrating communication between two wearable devices. Also, most of the HBC implementations suffer from the problem of signal leakage out of the body which enables communication even without direct contact with the body. In this article, we present BodyWire which uses an electro-quasistatic HBC (EQS-HBC) technique to enable communication between two wearable devices and also confine the signal to a very close proximity to the body. We characterize the human body channel loss under different environment (office desk, laboratory, and outdoors), posture, and body location conditions to ascertain the effect of each of these on the overall channel loss. The measurement results show that the channel loss varies within a range of $15 \mathrm{~dB}$ across all different posture, environmental conditions, and body location variation, illustrating the dynamic range of the signal available at the input of any receiver. Leakage measurements are also carried out from the devices to show the distance over which the signal is available away from the body to illustrate the security aspect of $\mathrm{HBC}$ and show its effect on the channel loss measurements. For the first time, a throughbody interhuman channel loss characterization is presented. Finally, a demonstration of secure interhuman information exchange between two battery-operated wearable devices is shown through the BodyWire prototype, which shows the smallest form factor HBC demonstration according to the authors' best knowledge.

Keywords: human body communication, human computer interaction, $\mathrm{HBC}-\mathrm{HCl}$, selectivity, security, wearable-wearable $\mathrm{HBC}$, electro-quasistatic HBC 


\section{INTRODUCTION}

Improvements in the solid-state circuit design over the last several decades have enabled the widespread proliferation of wearable devices and personal computing resources to a diverse number of users. Many daily functions such as financial transactions, SMS, health monitoring, and telecommunication have either been off-loaded to these devices or assessed as necessary features to carry everywhere. Because of this phenomenon, technology and interfaces that support the usability and interactions between these wearable devices continue to become more important from both a security and a usability perspective. Electronic communication between these devices is an essential feature for the functionality of these phones, smartwatches, etc., whether that comes in the form of secure key exchange between two devices or SMS data/ notification from a mobile phone to a smartwatch. Traditionally, these devices utilize Bluetooth to form this communication channel. In this article, we present an alternative communication using BodyWire, a single-wire communication technique that enables secure covert communication between wearable devices surrounding the body. BodyWire uses electro-quasistatic human body communication (EQS-HBC) accompanied with several critical design techniques that enable biological- and environmentalindependent operation for mobile wearables. EQS-HBC enables communication through the conductive properties of the human body and uses the body as a wire while limiting the physical signal leakage out of the body by operating at the electroquasistatic (lower frequency) regime. First, this enhances the security of ubiquitous wearables by eliminating the access of the physical signal from a nearby malicious attacker. This may seem like a small concern with software encryption, but researchers have demonstrated the hacking of critical lifesaving medical devices (Morgan et al., 2021; Shin and Lipton, 2018) through these encrypted radio networks. Second, it enables communication initiated through touch alone, eliminating the complicated setup process completely. Considering the growing number of ubiquitous devices surrounding the human body, this is an important aspect of keeping high accessibility and usability for a diverse group of users. BodyWire creates an intuitive interface of touch that every human is familiar with and can use. Simple touch is now augmented with the ability of electronic data transfer through a small form factor device that can be adorned on the human body to another worn device or any number of machines/terminals that we interact with today.

In this article, we explore the different environmental and human factors that affect the communication between two wearable devices during communication through HBC. The transmitter and the receiver device has been designed following techniques such as capacitive excitation into the body, high impedance capacitive termination at the receiver, and voltage-mode operation to enable its operation through EQS-HBC. Experiments are carried out in both the throughbody interhuman and intra-body scenarios over 2 months to assess the effect of different factors on the overall channel loss and ensure repeatable measurement results. We identify the key factors such as the surrounding environment and human body postures that affect the channel loss and determine it under varying conditions to quantify the effect of those individual factors. We also identify the amount of signal leakage out of the body at different distances and identify the range of distances over which channel loss measurements can potentially be affected by the signal leakage.

The rest of the article is organized as follows: the Methods section begins with human body communication background which provides a background of the HBC technology and compares the EQS-HBC implementation of this article with previous implementations. Next, the Key Design Techniques section describes the hardware considerations that enable the EQS-HBC device design in addition to Experimental Measurement Control Variables which discusses the key experimental constraints that need to be maintained to ensure repeatability of the experiments. The Results section contains Intra-body and Through-Body Inter-Human Body Communication (TBI-HBC) Measurements followed by a demonstration of the smallest ever form-factor EQS-HBC device.

\section{MATERIALS AND METHODS}

BodyWire uses human body communication (HBC), also commonly known as body channel communication (BCC), as the underlying communication method between the devices. HBC has been a field of active research over the last 2 decades since it was proposed by Zimmerman (1996) for personal area networks (PANs). It was proposed to capacitively couple picoamps current into the body to communicate between devices around it. Since then, there have been multiple aspects of HBC that have been explored, namely the channel characteristics of the human body, integrated circuit design specific for HBC, and complete system design demonstrating information exchange between devices. In this section, we provide a brief overview of $\mathrm{HBC}$ on these different aspects and identify the key limitations present in modern day HBC, highlight the possible solutions to alleviate those, and enable the demonstration of information exchange between appropriately ground-isolated wearable devices.

One of the critical elements in any HBC system is the channel characteristics provided by the human body. However, measuring the body channel characteristics without affecting it requires a careful experimental design with sufficient isolation between the ground of the measurement instruments and the measured devices. As a result, there is a wide variation in the measured channel response available in the literature such as in Ruiz and Shimamoto, (2005), Cho et al., (2007), Lučev et al., (2011), Bae et al., (2012a), Callejón et al., (2013), Hwang et al., (2016), and Park et al., (2017). Some of the measurements show low channel loss (around $20 \mathrm{~dB}$ ) due to improper ground isolation such as in the work of Ruiz and Shimamoto, (2005), as a result of which the wearable device gets a larger ground than a practical wearable device. HBC systems, which are designed based on these measurements, will not have the sufficient sensitivity to function in an actual scenario where the channel loss is 
significantly higher. A separate group of measurements from Lučev et al., (2011), Bae et al., (2012a), and Cho et al., (2007) shows the channel loss to be significantly high $(>90 \mathrm{~dB})$ at low frequencies $(<10 \mathrm{MHz}$ ), showing that frequencies $>10 \mathrm{MHz}$ is the optimum frequency for designing HBC systems. However, this limits these systems in terms of security and selectivity, as there is significant signal leakage out of the body. We will discuss in detail about the signal leakage aspects in the later sections. However, this shows the importance of proper channel characterization for the design of an HBC system. We have characterized the human body channel with proper ground isolation and termination at the receiver end to establish it as a broadband channel and utilize the measurements to design the BodyWire prototype. We discuss about the measurement setup and the corresponding results in detail in the following sections.

There have also been multiple studies for designing integrated circuits specific for HBC applications (Cho et al., 2009; Bae et al., 2012b; Cho et al., 2015; Park and Mercier, 2015; Saadeh et al., 2017; Maity et al., 2018). The relatively low loss of the broadband human body channel makes it possible to design energy-efficient circuits compared to that of traditional wireless circuits such as Bluetooth. However, the focus of this article is on the commercial off-the-shelf (COTS) component-based system design for the demonstration of HBC. There have been multiple studies for the system-level demonstration of HBC. The demonstrations primarily show communication between a wearable and an Earth ground-connected device. Moreover, these demonstrations suffer from the problem of signal leakage out of the devices even when the person is not in direct physical contact with it. This makes the interactions not selective and the data transmission prone to attacks from an unintended recipient. One of the earliest commercial implementations of HBC is through Microchip Technology's BodyCom system (Brain Bailey Microchip Technology Inc, 2014). They show the unlocking of car or home doors using a wearable transmitter device; however, the datasheet reveals that the loss is high at low frequencies, and large voltages $(75 \mathrm{~V})$ and large coupling devices (door frames/metal surfaces) are required rendering a wearableto-wearable implementation impossible. The devices communicate even with the transmitter in the user's pocket showing no strict requirement of direct contact with the body for communication. Author Anonymous (2021) shows the use of a ring as an intermediate data transfer device for information exchange between two computers. There are demonstrations shown in ikinamo, (2009) where an image gets transferred from a mobile transmitter to a wall-connected device. However, from the demonstrations, we can clearly see information getting transferred between the devices even when the user is far away from the receiver and only holding the transmitter device. Williams (2021) shows transfer of color information from a wrist watch to the clothing of another person when the persons come in contact with each other. The authors of Varga et al., (2018a) show the example of a game where the users communicate with floor tiles with a wearable watch-like device. The devices can communicate even when the user is wearing a footwear showing communication without direct physical contact with the devices and the potential for signal leakage. As a follow-up work (Varga et al., 2018b), the authors provide SNR measurements for different intra-body and through-body interhuman scenarios for communication between wearable devices and provide guidelines for the design of groundless BCC systems. As a demonstration, the authors show an application of music streaming through BCC headphones, operating at a carrier frequency of $8 \mathrm{MHz}$, which can again potentially suffer from signal leakage out of the body. The authors in Hessar et al., (2016) re-purpose the finger print sensors of commodity devices such as smartphones for sending signals through the body for authentication purposes at a data rate of $100 \mathrm{~s}$ of bits per second. Finally the authors of Maity et al., (2020) demonstrate an EQS-HBC implementation, however, is only capable of intra-human communication (between one person). There are other implementations, such as TAP (Park et al., 2006), Earthlings Attack! (Takahashi et al., 2011), Wearable Key (Matsushita et al., 2000), and CarpetLAN (Fukumoto and Shinagawa, 2005), which use human as the communication medium for interaction between devices. Through BodyWire, we show the first implementation of physically secure interhuman information transfer, utilizing channel loss results obtained from thorough characterization of the effect of different factors. The associated challenges and comparison with state of the art are shown in Table $\mathbf{1}$.

In a broader sense, HBC can be classified as one of those human computer interaction techniques that enable interaction between devices through touch using electrical signal transmission through the body. There are multiple studies which identify either single/multiple touches on a screen such as Field mice (Smith, 1996), Smartskin (Rekimoto, 2002), Diamond Touch (Dietz and Leigh, 2001), Swiss-Cheese Extended (Grosse-Puppendahl et al., 2013), OpenCapSense (Lan et al., 2017), DGTS (Habib et al., 2009), and Tile Track (Valtonen et al., 2009). BodyWire enables communication between devices only when they are in direct touch contact with the body but does not provide the capability to find the location of the touch. SkinTrack (Zhang et al., 2016), AuraSense (Zhou et al., 2016), High5 (Kim et al., 2014), Living Wall (Buechley et al., 2010), and iSphere (Lee and Selker, 2004) enable gesture recognition using interaction between the electric field and the human body.

\subsection{Key Design Techniques}

\subsubsection{Capacitive vs. Galvanic Coupling}

The theory of operation behind capacitive coupling (Zimmerman, 1996) (Figure 1A) HBC can be understood by the use of traditionally parasitic capacitances as the return path for what would otherwise be a traditional wireline channel. On the other hand, galvanic coupling (Wegmueller et al., 2010) (Figure 1B) of the signal to the body involves applying a differential excitation where the signal path is localized in the human.

There are two key reasons for choosing capacitive coupling for a fully wearable application over galvanic coupling. First, capacitive coupling introduces much less current in the body, and hence makes it more attractive from a safety perspective, as the current is heavily limited by the return path capacitances. The more important reason is that since the communication utilizes 
TABLE 1 | Comparison of BodyWire with state-of-the-art HBC implementations.

\section{Metric}

$$
\begin{gathered}
\text { Author Anonymous (2021), } \\
\text { Williams (2021), } \\
\text { Varga et al. (2018a), } \\
\text { Varga et al. (2018b), } \\
\text { Hessar et al. (2016), } \\
\text { Park et al. (2006), } \\
\text { Takahashi et al. (2011), } \\
\text { and Fukumoto and } \\
\text { Shinagawa (2005) }
\end{gathered}
$$

Wearable-to-wearable HBC demonstration

High loss at low frequency and frequency dependent due to improper impedance termination (resistive)

Electro quasi-static operation

Negative effect of distance on operation

Size of the device

Capacitance division at $\mathrm{Rx}$

Required excitation voltage

Interhuman communication

No
Various
No
Various
Large
Various
Various
No Varga et al. (2018b). A few
static measurements

No
No
Yes
No
$5 \times 6 \mathrm{~cm}^{2}$
Yes
$3.3 \mathrm{~V}$
No

No, Base Unit (Machine) to Mobile (Wearable) $\rightarrow$ Easier Channel

Yes, as evident in AN1391 Page 1 description“. . .signals are more attenuated at lower frequencies than at higher frequencies"

EQS frequency range but no wearable-wearable operation and no analysis of physical security

Yes, as evident in AN1391 Page 4 Section "Relationship to a Common Reference"

"Large central conductor such as a door frame or metal desk"-AN1391 Page 4

No

$75 \mathrm{~V}$

No
This work

$\begin{array}{ccc}\text { Maity } & \text { Saadeh } & \text { This } \\ \text { et al. } & \text { et al. (2017) BodyCom } & \text { work } \\ (2020) & & \end{array}$

measurements

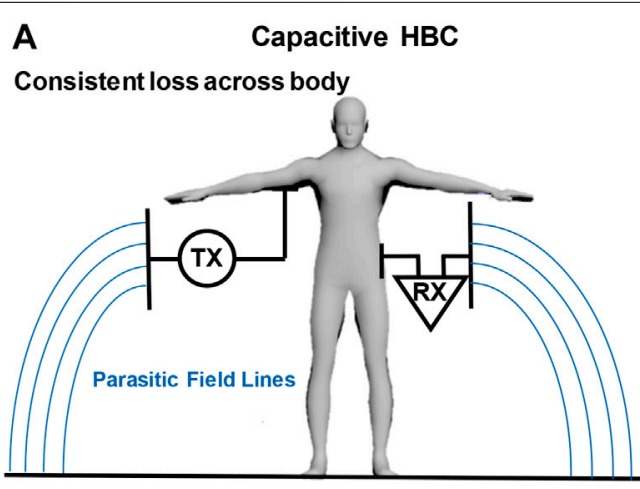

B Higher loss away from TX

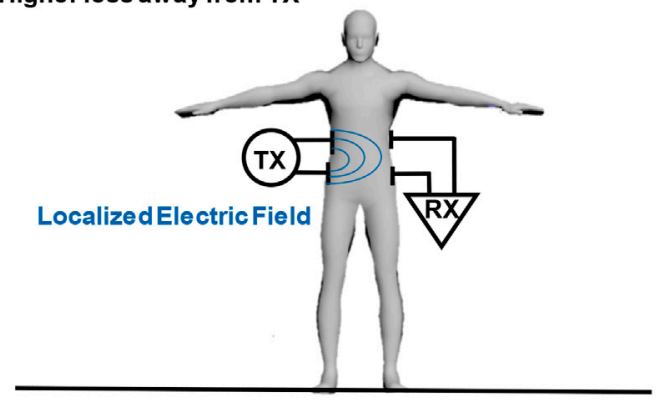

FIGURE 1 | Human body communication using (A) capacitive coupling mode versus wearable devices as the loss is consistent across the entire body.

capacitance between the devices to ground and this capacitance (few $\mathrm{pF}$ ) has significantly higher impedance than the rest of the closed loop path, most of the signal loss occurs across this parasitic capacitance. Hence, the received signal strength across different parts of the body is almost equal. In later sections, we carry out experiments to highlight this particular benefit of capacitive coupling.

\subsubsection{High-Impedance Termination}

Proper termination at the receiver end is another key factor in determining the overall channel loss between the transmitter and receiver devices. A simple circuit theoretic analysis of the $\mathrm{HBC}$ channel model shows that the overall channel loss will reduce if a high-impedance termination is used at the receiver end. Similarly, a capacitive termination at the receiver end will reduce the lowfrequency channel loss and make the channel loss almost independent of frequency. A detailed theoretical analysis and experimental results of the channel loss based on a biophysical model can be found in Maity et al., (2019). The HBC receiver circuit needs to be designed taking the constraint of highimpedance capacitive termination into account to enable lowloss low-frequency operation.

\subsubsection{Electro-Quasistatic Human Body Communication}

The high-impedance capacitive termination at the receiver end reduces the human body channel loss at low frequencies and extends the frequency range of its operation. This opens up a new 
frequency band of operation in the sub- $\mathrm{MHz}$ range, which was previously deemed unusable due to the high channel loss reported in the previous literature. We utilize this low-frequency band to enable electro-quasistatic human body communication (EQSHBC) and use it for our demonstrations. In EQS-HBC, the wavelength of the transmitted signals is significantly larger than the size of the human body, as a result of which the signal is constant across the body. This also helps reduce the leakage out of the body significantly as the signal leakage is proportional to the frequency of the transmitted signal (Das et al., 2019). The low leakage helps in characterizing the human body channel with minimal effect from inter-device coupling, which can provide optimistic channel loss measurements. It also enhances the security of the HBC devices as the signal is confined within a small distance around the body and reduces the probability of an unintended attacker snooping the signal.

\subsubsection{Voltage-Mode Operation}

In the design of the BodyWire system, the devices send signals between the transmitter and the receiver with the goal of maximizing efficiency instead of traditional RF designs which maximize power transfer. Using voltage for information transfer benefits from the high-impedance capacitive termination at the receiver as it maximizes the amount of voltage at the receiver end. Maximizing the power at the receiver requires different design constraints for optimization compared to voltage-mode operation, which makes the design of the current receiver different compared to that of the earlier $\mathrm{HBC}$ implementations. From a circuit design perspective, maximization of power transfer requires the impedance at the receiver end to be matched with the source. However, since the body channel itself has high impedance-the transmitter system would have to be designed with high impedance-this is contradictory to the fact that the source impedance needs to be minimized for transfer to be efficient. Since the frequency is low, matching is not necessary as reflections are not of concern. Hence, the voltage-mode circuit design is better suited for the operation of wearable-wearable HBC systems, where the received signal is low due to ground isolation.

\subsubsection{Frequency of Operation}

From the previous literature presented by Yang et al., (2021), there is interference on the human body both at $60 \mathrm{~Hz}$ due to the mainline and $45-50 \mathrm{kHz}$ due to CLF Lamps as well as their harmonics. As a result, operating at a higher frequency far and away from these strong interferences enables a better front-end implementation (filtering). Second, as the target data rate for comparable devices is typically in the 10's of kbps-any higher carrier frequency in the EQS regime would only result in higher power consumption with no significant benefits unlike the RF design where frequency has a high impact on channel loss properties and optimal transceiver design.

\subsection{Experimental Measurement Control Variables}

As described previously, wearable-to-wearable human body communication is challenging to design due to the variations in channel loss due to the nature of capacitive-mode $\mathrm{HBC}$ systems. However, if the capacitive termination voltage mode technique is utilized properly, these variations can be measured and quantified into manageable design constraints for any wearable human body communication system. This section will explain the custom experimental setups (Figure 2) and control variables required to carry out these measurements. We consider five main design factors when attempting to design repeatable and controlled wearable-to-wearable HBC experiments. These include 1) proper Earth ground isolation, 2) device size, 3) signal leakage, 4) device-to-body ground isolation, and 5) environment-induced variations.

\subsubsection{Earth Ground Isolation}

The most important control factor for wearable-wearable HBC is proper ground isolation on both the transmitter and receiver devices. As actual wearable systems will have ground isolation from Earth ground, the measurement equipment must also have complete ground isolation. Therefore, the use of any measurement device requiring a building power supply would result in inaccurate measurements as one would be boosting the signal strength by introducing a much more convenient return path between the devices through the measurement equipment. To achieve proper ground isolation, a custom-printed circuit board in wearable form factor including a microcontroller with 12-bit ADC, embedded Bluetooth module, and signal conditioning analog front-end was fabricated. This enables the experimental data to be accumulated in a wearable form factor and transmitted wirelessly to a laptop for post-processing. The Bluetooth module transmits the sampled received human body communication signal to a computer serially, and the channel loss is calculated taking into account of the gains and losses introduced by the measurement setup, as shown in Figure 2. This process eliminates any Earth ground from affecting the channel loss numbers. A $3.7 \mathrm{~V}$ transmit signal is coupled onto the body through a printed circuit board at $415 \mathrm{KHz}$ and measured at a high-impedance termination receiver that has the same analog signal front-end as the final demonstration shown through the handshake example.

\subsubsection{Device Size and Packaging}

The size of the transceiver and receiver printed circuit boards with their wearable packaging is $4 \times 4 \mathrm{~cm}$ and is coupled to the body through a $2 \times 3 \mathrm{~cm}$ electrode which is secured by a wearable band as shown in Figure 3. The device size must be strictly controlled in order for the experiments to be repeatable. This phenomenon can be explained using the theory of operation for capacitive voltage-mode HBC. Increasing the size of the device gives the return path capacitance a larger value and hence reduces the loss in the channel. A dangling component or a longer connecting wire can result in a change in the return path capacitance and hence change the measurement result. So, it is important to ensure that the device is packaged identically (no loose components, minimal size wire for connection) between different measurement sessions across multiple days. Similarly, the environment around the device is also an important factor in determining the return path capacitance in a capacitive HBC scenario. Hence, it is necessary to keep the environment around the person identical for measurements corresponding to the same 


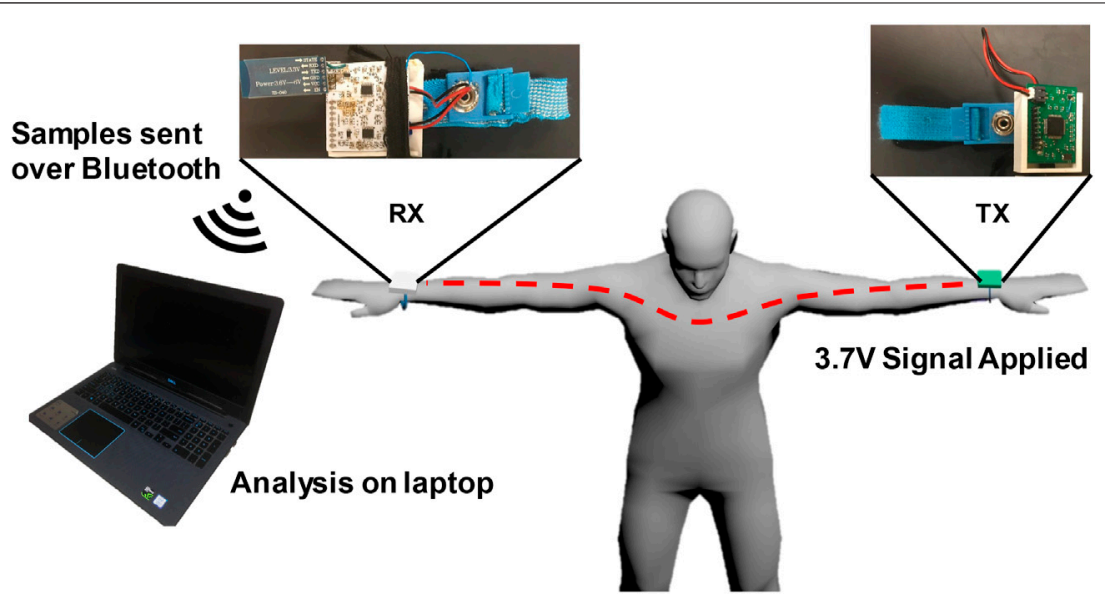

FIGURE 2| Experimental setup for wearable-to-wearable channel loss measurements. The transmitted signal is received and sampled using an ADC, which is sent over Bluetooth to a laptop for analysis and channel loss calculations. This ensures that the measurements are not perturbed by a strongly ground-connected device, which results in optimistic channel loss results.

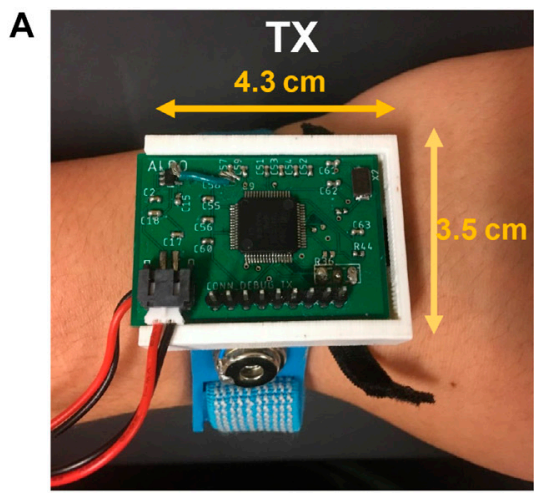

B

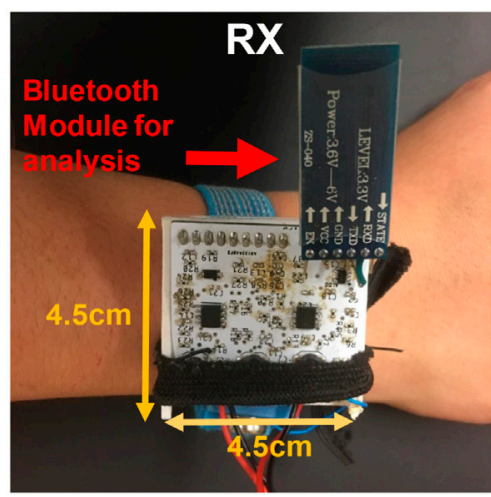

FIGURE 3 | Image of the measurement setup boards with dimensions. (A) TX board features a form factor of 43 by $35 \mathrm{~cm}$, and (B) RX board features a form factor of 45 by $45 \mathrm{~cm}$ with a Bluetooth module that transmits the sampled ADC values for analysis.

experiment across multiple days and subjects. This not only ensures repeatability of results but also enables us to quantify the effect of each factor on the overall channel loss.

\subsubsection{Signal Leakage}

Leakage from circuit components can affect HBC channel measurement results through two primary methods. The first is improper shielding of the signal being applied to the body. Electrical wires between the body and the device that are not properly shielded will act as a source of leakage for the HBC signal and hence would result in inaccurate loss measurements that are optimistic for packaged wearables. It is obvious that this effect is not an important factor for some applications, but in order to achieve the security and low leakage of EQS-HBC, this is an important consideration. The second method is improper packaging of the battery. Allowing the battery to be exposed enables ground coupling between the TX and RX which gives lower channel loss. Hence, proper wire shielding and battery packaging are required to set up fundamental $\mathrm{HBC}$ measurements, so that the measured channel loss is not affected too much by the signal leakage. Moreover, this also reduces the security aspect of $\mathrm{HBC}$ systems as the signal can be snooped by an attacker at distances far away from the body.

\subsubsection{Device Ground-to-Body Isolation}

It is necessary to ensure appropriate isolation between the device ground and human body to ensure capacitive excitation of signals into the body. If there is direct connection between the device ground plane and the skin or close proximity of the ground plane to the body $(<2-3 \mathrm{~mm})$, it will effectively result in galvanic operation as the excitation/termination will be differential. There will be a local loop formed through the body between the signal and ground terminal of the device, where most of the signal will be localized. This effect is observed to be much larger with the battery specifically, since it forms part of the ground plane. Improper device-to- 
body ground isolation would shunt the signal away from the intended receiver either on a different user or the other side of the body. Effectively, the wearable device's signal and ground planes are being electrically shorted through a small coupling formed between the TX device ground and the body. This isolation must be present in order for fully wearable HBC to be functional. Hence, it is important to package the device ensuring that no part of the ground plane is directly touching the body. This is a key constraint in the design of any wearable capacitive $\mathrm{HBC}$ device.

\subsubsection{Environmental Control}

The environment around the body also has an important role in determining the overall channel loss, since the return path capacitance between the transmitter and the receiver is dependent on the coupling of the devices with the environment. As already mentioned, the return path capacitance plays an important role in determining the overall loss between the transmitter and receiver. As part of the characterization process, we carry out measurements in different environments such as in an office, lab, and outdoors. It is essential to ensure that the environment remains the same among different set of measurements carried out across multiple days and on multiple subjects. This requires a careful design of the experiments to ensure repeatability of the setup.

\section{RESULTS AND DISCUSSION}

\subsection{Intra-Body Channel Loss}

This section will explore the various experiments performed in order to identify the different factors that affect channel loss in a wearable-wearable HBC scenario and help us realize the fully wearable $\mathrm{HBC}$ communication device. Following are the different sets of experiments that are carried out for wearable-wearable HBC characterization: 1) channel loss on multiple users, 2) interdevice coupling, 3) intra-body posture and environmental dependency, 4) channel loss across various body locations, and 5) electro-quasistatic leakage measurements. To ensure proper ground isolation and true wearable-to-wearable measurements, the signal received at the receiver end is sampled through an ADC and transmitted over Bluetooth to a computer for further analysis and loss characterization as shown in Figure 2.

\subsubsection{Channel Loss on Multiple Users}

The channel loss study on multiple users is necessary to ensure a design that is robust to biological variations between users. The theory of operation for the wearable-wearable HBC device is inspired from the biophysical circuit model presented from Maity et al., (2019). The parameters of the body are characterized in order to ensure that wearable-wearable $\mathrm{HBC}$ is possible to realize. The variations in channel loss across users are important to ascertain the range of signals (dynamic range) that is expected at the input of the receiver device. The dynamic range plays an important role in determining the design complexity of the receiver front-end circuit. Subjects are measured for channel loss at different times of the day over a period of 2 months. This experiment covers subjects that have different physical parameters (age, height, weight, sex, etc.) and provides a sample of channel loss that designers of fully wearable HBC systems must anticipate. These measurements can be used to develop a signal conditioning front-end that enables reliable communication through this technology. Channel loss for multiple users is measured in a laboratory environment. Other known factors that affect the return path such as posture, environmental coupling, and device package positions are kept strictly controlled and in order to provide an accurate understanding of the isolated effect of different user parameters on this device's channel loss.

The results of the channel loss across multiple subjects are plotted in Figure 4A. Channel loss for the fully wearable HBC system falls within a range of $69 \mathrm{~dB}-75 \mathrm{~dB}$. These numbers present a worst case number for most applications, given the minimal ground size of the devices used to carry out the experiments in this article.

\subsubsection{Inter-Device Coupling}

Inter-device coupling describes the interaction in which the ground planes of the transmitter and receiver devices are coupled. This enables the return path of communication to be between the coupling of the device rather than the ambient parasitic environment. This coupling dominates the return path and thus the channel loss at close distances. This theory is established and modeled by the authors of Datta et al., (2021). However, wearable device measurements are not provided-in this article, we analyze the effects of inter-device coupling and body shadowing on multiple subjects.

The inter-device coupling is measured by bringing the transmitter device very close to the receiver without any direct contact and measuring the received signal. This is repeated over multiple distances up to $25 \mathrm{~cm}$, beyond which the received signal falls below the receiver's sensitivity. The measurement results in Figure 4B show that the inter-device coupling loss can be lower than the channel loss through the body for distances up to $10 \mathrm{~cm}$. This shows that intra-body channel loss measurements for channel lengths less than $10 \mathrm{~cm}$ can be heavily affected by the inter-device coupling rather than the actual body channel loss. Hence, any measurements characterizing body channel loss should be carried out for channel lengths $>10 \mathrm{~cm}$.

\subsection{Intra-Body Posture and Environmental Dependency}

Practical implementations of wearable HBC devices require the communication through the body to be functional irrespective of environment and posture variations. So, the devices need to be designed to be able to tolerate the variation introduced by user posture and surrounding environmental effects. Posture varies channel loss primarily from the geometric positioning of the device ground plane with respect to Earth ground, which varies the capacitance in the return path similar to the size of the device and battery positioning. Similarly, having many physical objects 

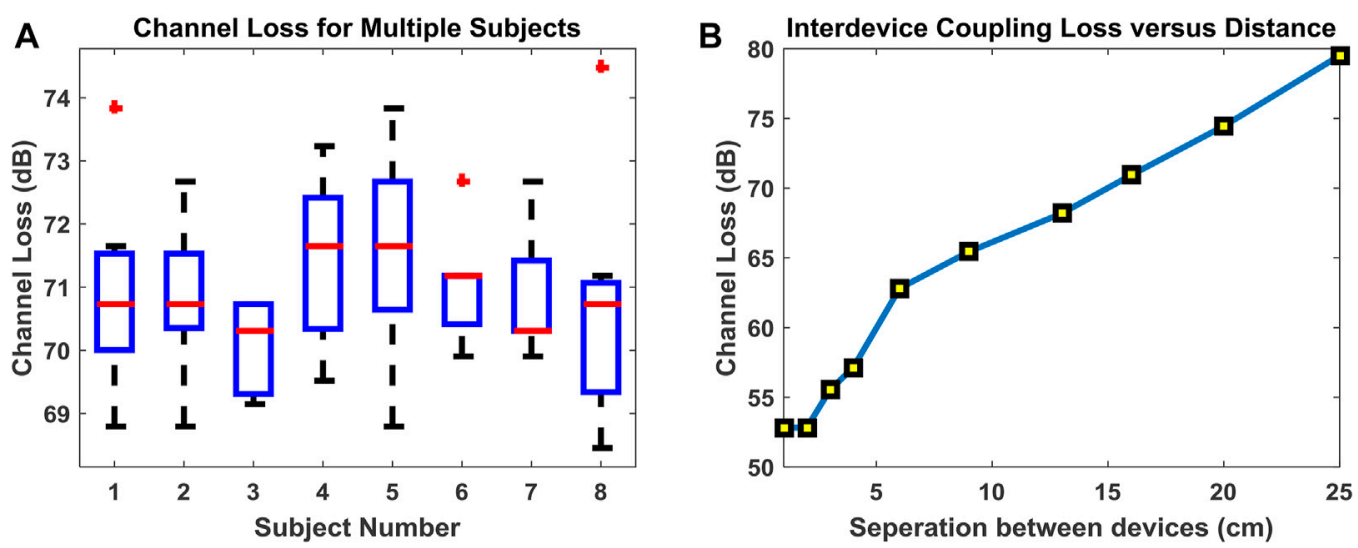

FIGURE 4 | (A) Box and whisker plots of channel loss for eight users over 2 months. The range of loss falls between 68 and 75 dB. (B) Inter-device coupling measurements without the body show that the communication happens mostly between the two devices for channel lengths less than $10 \mathrm{~cm}$.

in the environment can introduce additional signals or ground planes depending on user contact or proximity. These objects can potentially affect the path of transmission which in turn changes the loss introduced by the capacitive voltage division between the capacitance of the body and that of the environmental surroundings. For example, if the experiments for channel loss were performed with both the receiver and transmitter over a large table, the capacitance formed between the devices' return path is varied as the return path is now both through the table, when otherwise the field lines would have terminated to the floor. However, the behavior of this coupling is very difficult to predict in theory due to the slight changes in conductivity and positioning between every environment and posture. Like the multi-user channel loss setup, the goal for these series of measurements is to determine if a dynamic range problem exists for the signal conditioning front-end. The reason the design of the experiments for environmental and posture studies was coupled is due to the close nature of their respective effects on the channel loss as a phenomenon of physical device placement and geometry. Also, the experimental setup was designed to ensure that they stay consistent across multiple days and users for repeatable measurements and proper attribution of the effect of different factors on the channel loss.

\subsubsection{Posture Results}

The user posture experiments are carried out with the transmitter and receiver devices fixed onto the subject's wrists. From this point, two separate characterizations are performed; one where the user's arm moves horizontally and the other where the user's arms move vertically with respect to the body. Figures $\mathbf{5 A}, \mathbf{C}, \mathbf{E}$ and Figure 7A give an overview of the positions of the user's arm during the horizontal and the vertical experiments, respectively. These measurements provide interesting insight into the postures and fundamental effects that vary channel loss. For control, all posture and environmental studies are completed on the same subjects over similar times of the day. There are two main driving forces that affect channel loss for the horizontal posture: 1) shunt capacitance as a result of body shadowing and 2) inter-device coupling. As observed in any of the posture plots in Figures 6A-C, the loss is driven up as the devices move inward closer to the core of the body (position moves from 1 to 3 ). This is due to the effect of coupling between the central/lower parts of the body surfacing. This capacitance shunts the signal away from the receiver to the ground plane. Since the subject is standing on the floor, the body capacitance inevitably shunts some signal away from the receiver, thus increasing loss. As the arm moves closer to the center/core of the body, the channel loss increases. The trend described previously is dominant over the trend of channel loss until the devices are close together (approx. $<10 \mathrm{~cm}$ ), at which point, the ground plane coupling of the transmitter and receiver device forms the dominant physical phenomenon in determining loss. The inter-device coupling can be observed in Figures 6D-F where as we move to the 4-4 position the loss is less. In the $4-4$ position, the device is touching the main part of the body, effectively short circuiting the shunt capacitance of the body, thus enabling the body to become the signal plane. It can be observed that the 3-3 position, which maintains both devices close to the center body but not touching, shows high loss as the signal is being shunted to the body and cannot be measured by the receiver. Furthermore, in Figures 6D-F, it can be seen that as the device moves closer (4-4 to 4-1 or 1-4 to 4-4), the channel loss decreases-illustrating the effect of inter-device coupling. Despite all the variations due to the two phenomena, the channel loss introduced by the posture is still tolerable for a system, showing a maximum variation of $6-7 \mathrm{~dB}$, suggesting that no significant dynamic range problem arises from variation in posture. The vertical posture study result in Figure $7 \mathbf{B}$ shows the inter-device coupling effect in the 5-5, 5-4, and 4-5 positions. Outside of the inter-device coupling, all other postures show the consistent average measurement result, giving the insight that vertical posture is not a factor that affects channel loss.

\subsubsection{Environmental Dependency Results}

The posture experiments are carried out in three different settings-a controlled lab environment, a desk setup, and an open outdoor area. The desk represents the best case scenario 

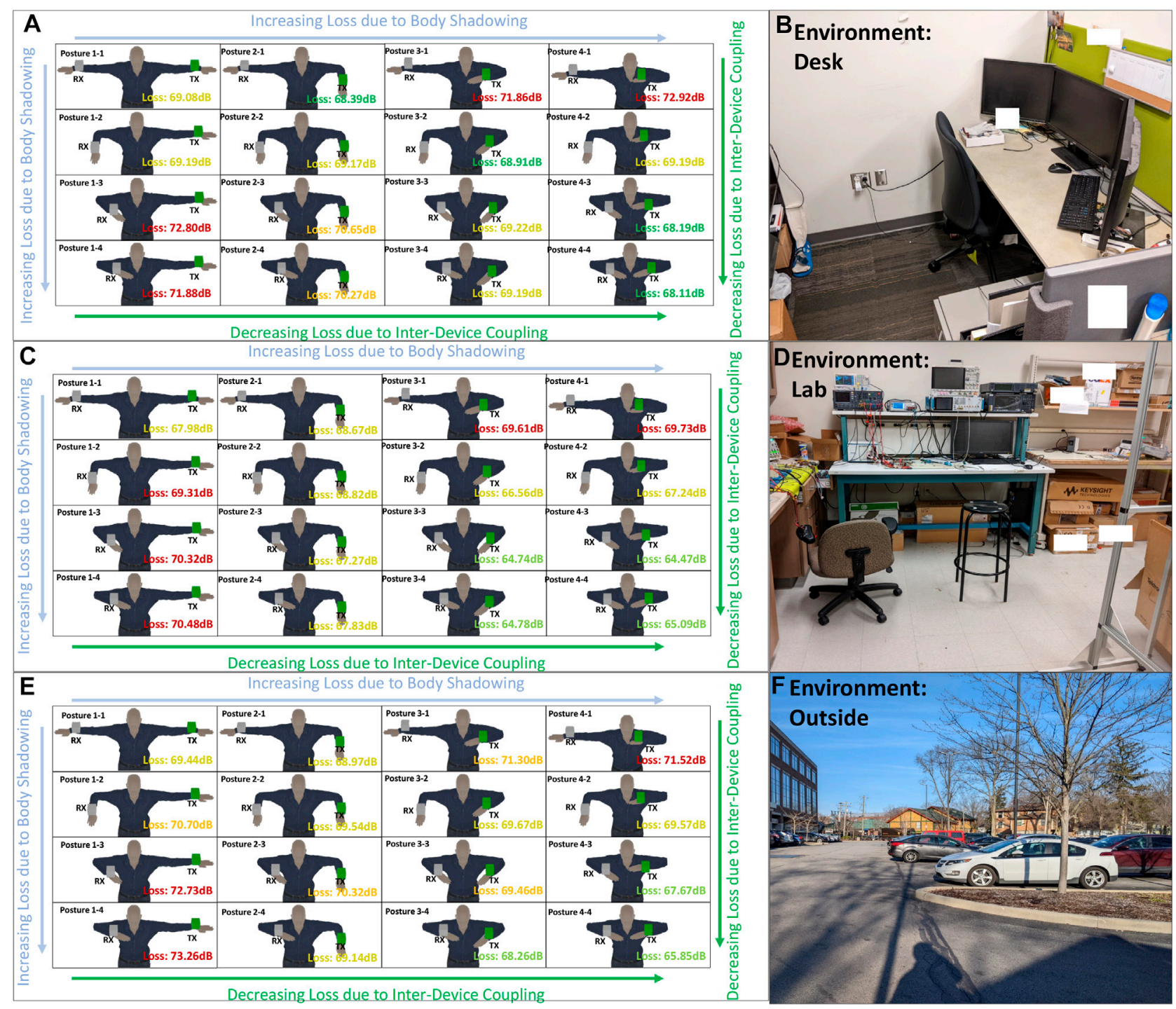

FIGURE 5 | Sample human model illustrating human arm postures. (A) Posture arm positions and loss 1-1 through 5-5 for the desk environment. (B) Desk environment in which the measurements were completed. (C) Posture arm positions and loss for 1-1 through 5-5 for the lab environment. (D) Lab environment in which the measurements were completed. (E) Posture arm positions and loss 1-1 through 5-5 for the outside environment. (F) Outside environment in which the measurements were completed.

for channel loss measurements as the return path has the assistance of the nearby chair and table. The user is sitting in a chair, and the devices are over a table. The outdoor measurements represent a worst case scenario, where there are no objects for the devices to couple within tens of meters. Hence, the return path capacitance will be minimum, thus increasing the channel loss. The motivation is to investigate the signal coupling differences between the devices in a sample of different environments and understand channel loss within these different environments. As can be seen in Figure 8, as the environment changes from a desk to a laboratory, the channel loss goes down across the board. This result is in line with theoretical expectations; since as the number of objects increases around the user, the distance to a terminating common ground is reduced. However, these objects can also act as agents that steal the signal away from the user-hence the loss could be higher than a complete open space-the mixed results can be explained by this phenomenon. Once again, these ambient objects increase the return path capacitance and thus reduce/ decrease the loss in the channel depending on orientation. Finally, the outdoor measurements give an extreme sample. Despite the variations observed in Figure 8, the average channel loss between the best and worst case samples vary only by $1.8 \mathrm{~dB}$, from 67.8 to $69.6 \mathrm{~dB}$ channel loss. This result confirms that environmental coupling has minimal effect on channel loss measurements and hence does not cause a significant dynamic range issue for the device designer. In order to characterize the distance up to which objects will be effectively coupling and 


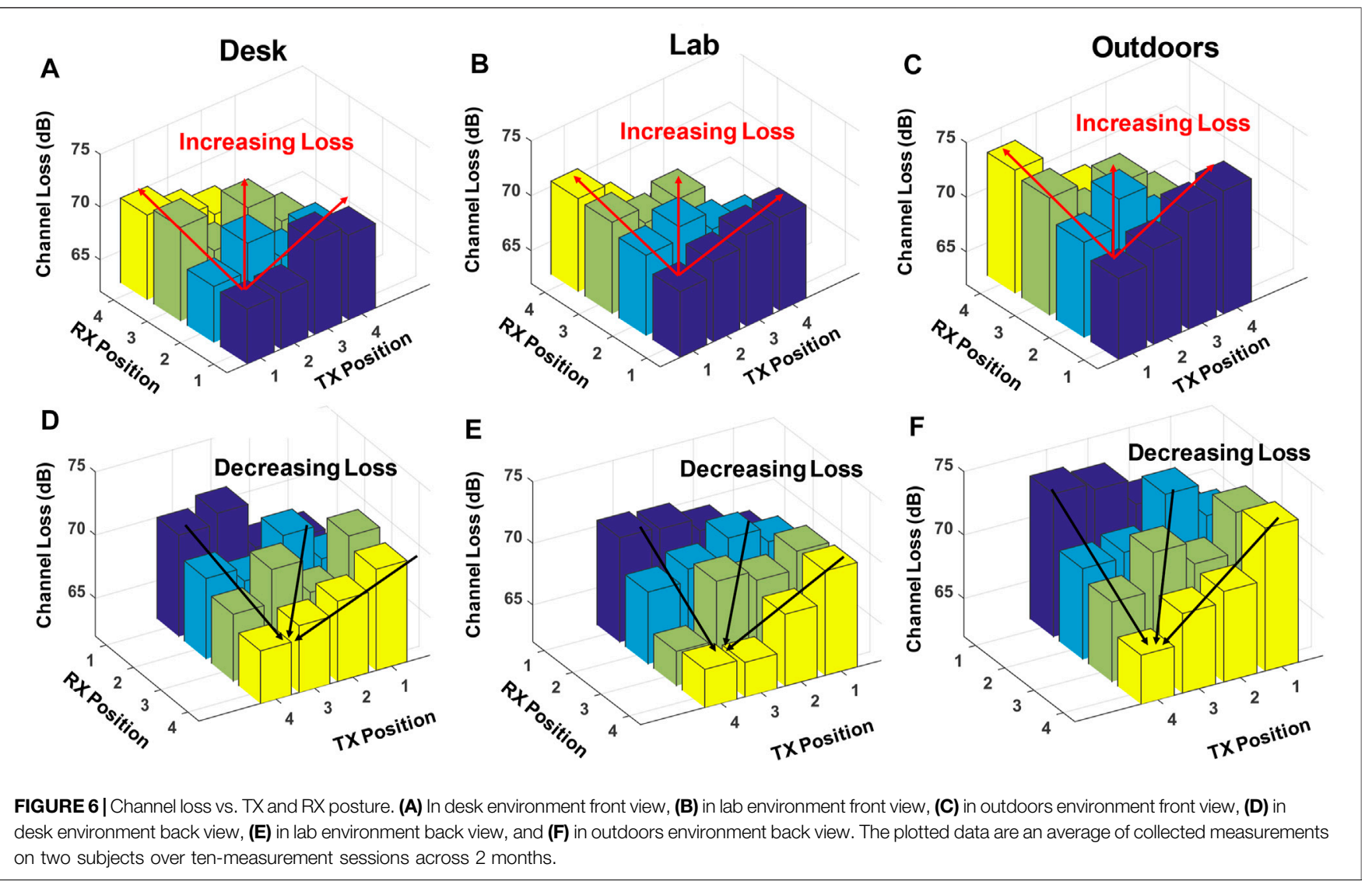

A

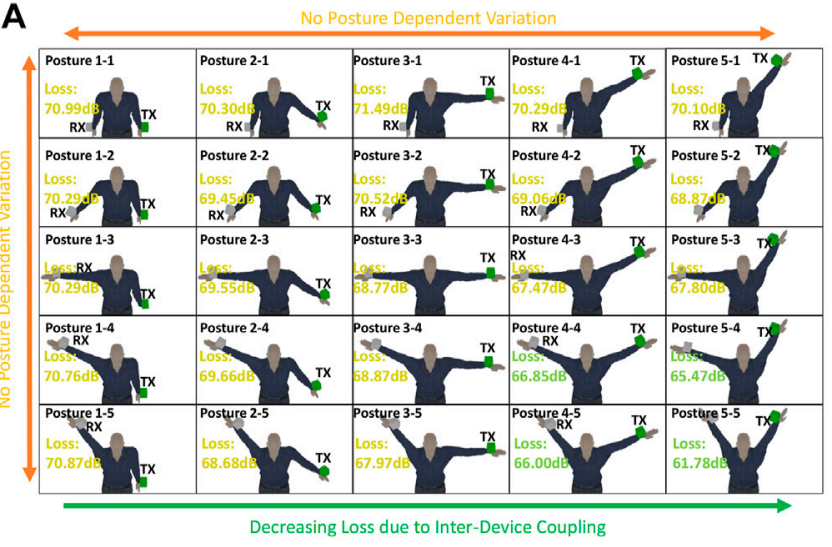

B Vertical Arm Posture Study

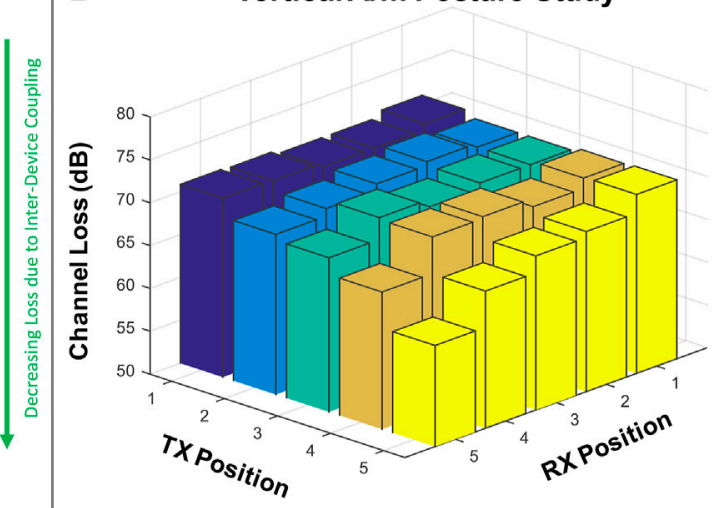

FIGURE 7 | (A) Human figures demonstrating vertical arm positions 1-5. (B) Channel loss versus the position of TX and RX arm. Channel loss is agnostic to the vertical position of arms with the exception of the lower loss due to inter-device coupling in $5-5,4-5$, and 5-4.

affecting the channel loss, an experiment targeted at measuring channel loss from a single isolated source of coupling is executed as shown in Figure 9A. The channel loss for a user is measured in a nominal posture at predefined distances away from a large coupling object. The averaged channel loss results are presented in Figure 9B. It can be seen that the coupling effect has approximately a $4 \mathrm{~dB}$ effect on the channel loss up to around $50 \mathrm{~cm}$. Beyond $50 \mathrm{~cm}$, the large coupling object poses little to no effect to the wearable-to-wearable communication. This shows that for enclosed indoor environments, the objects around the users can have a reducing effect on the channel loss. Outdoor environments provide a worst case scenario in 


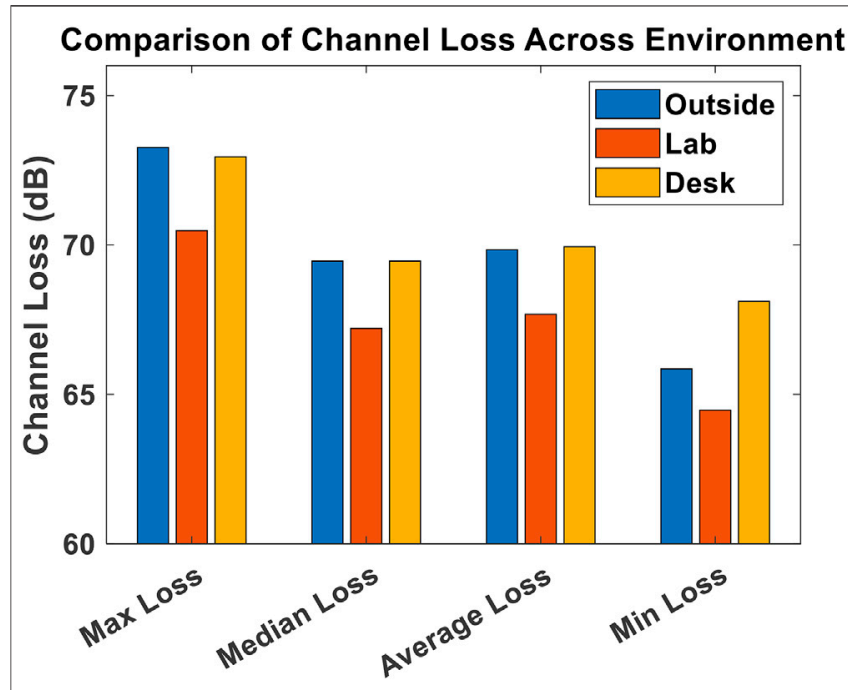

FIGURE 8 | Bar graph capturing maximum, average, median, and minimum channel loss for all environments for intra-human HBC.

terms of channel loss on average. However, this experiment again shows that the dynamic range is $4 \mathrm{~dB}$ for the indoor channel loss, not posing too strict a constraint on the receiver design.

\subsection{Channel Loss Across Various Body Locations}

As described in the overview of HBC, capacitive voltage HBC systems give uniform channel loss across all parts of the body. This effect is contrary to the galvanic approach results, in which the signal is localized between the transmitter and receiver. Galvanic coupling suffers for transmission across long lengths within the body, which degrades the usability of this technology significantly as you would only be able to transmit very short distances on the body. BodyWire implements capacitive-mode HBC which opens up this technology to many more applications.

This set of measurements is carried out to provide a characterization of the performance of capacitive voltage-mode HBC along different paths across the body. In one set of experiments, the transmitter device is moved across the arm keeping the receiver fixed on the other arm and vice versa (Figure 10A). For the second experiment, the transmitter is kept fixed on the left arm, while the receiver is moved across both legs as shown in Figure 10B. The final experiment keeps the transmitter fixed on the left leg and characterizes the loss along both arms as shown in Figure 10C. Channel loss is characterized in each of these six scenarios, and the variation between transmissions across different parts of the body is characterized as shown in Figures 10A-C. The results from the experiments show that the channel loss remains within a range of $3 \mathrm{~dB}$ across various channel lengths. This illustrates the advantage of constant loss across the body provided by capacitive $\mathrm{HBC}$.

\subsection{Electro-Quasistatic Leakage Measurements}

Currently, wireless technologies such as Bluetooth uses radio waves, which results in electromagnetic radiation up to several meters in every direction and results in the signal getting intercepted. BodyWire implements the technique of electroquasistatic human body communication, which aims to confine the communication signal near and within the body (Das et al., 2019). This technique prevents a potential malicious attacker from intercepting sensitive communications from far away and gives body area networks an additional layer of physical security.

Hence, an important characterization of any on-body communication between two wearable devices lies in determining the extent to which the signal is available away from the body. These experiment setups are depicted in Figure 11A. For Scenario 1 and Scenario 2, a human with a

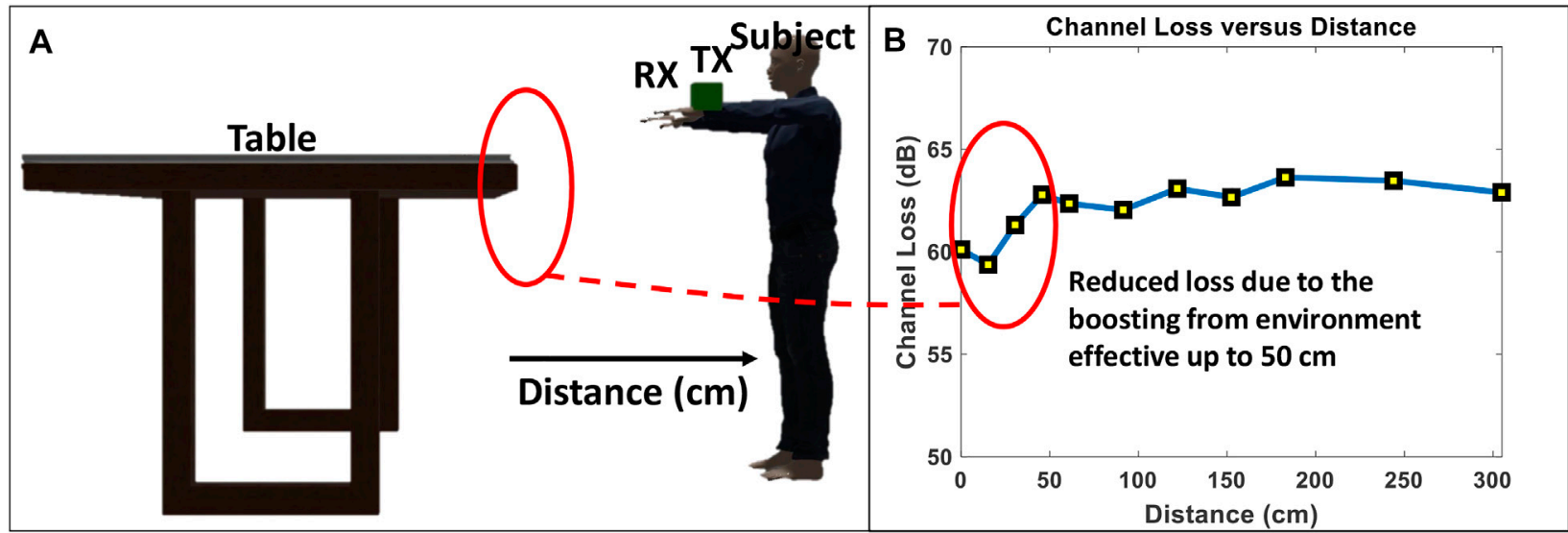

FIGURE 9 | (A) Experimental setup for the experiment illustrating the effect of the environment on channel loss. (B) After a distance of $50 \mathrm{~cm}$, the effect of the environment is not observable on the subject. 


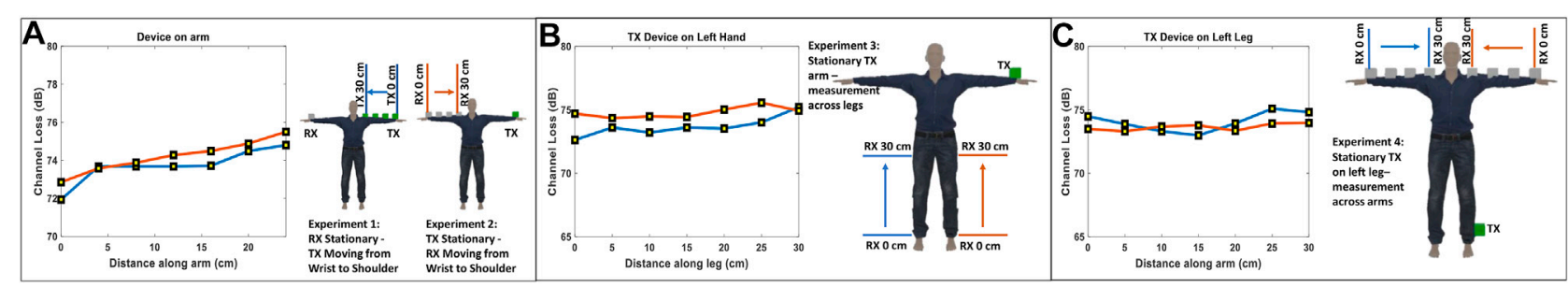

FIGURE 10 | Results of experiments performed to verify equal signal strength on the entire body. (A) Small $3 \mathrm{~dB}$ variation can be observed due to the devices moving toward the torso. (B) Loss is higher in general for the opposite leg but only around $3 \mathrm{~dB}$. (C) Loss is similar for both arms showing no difference in channel loss.

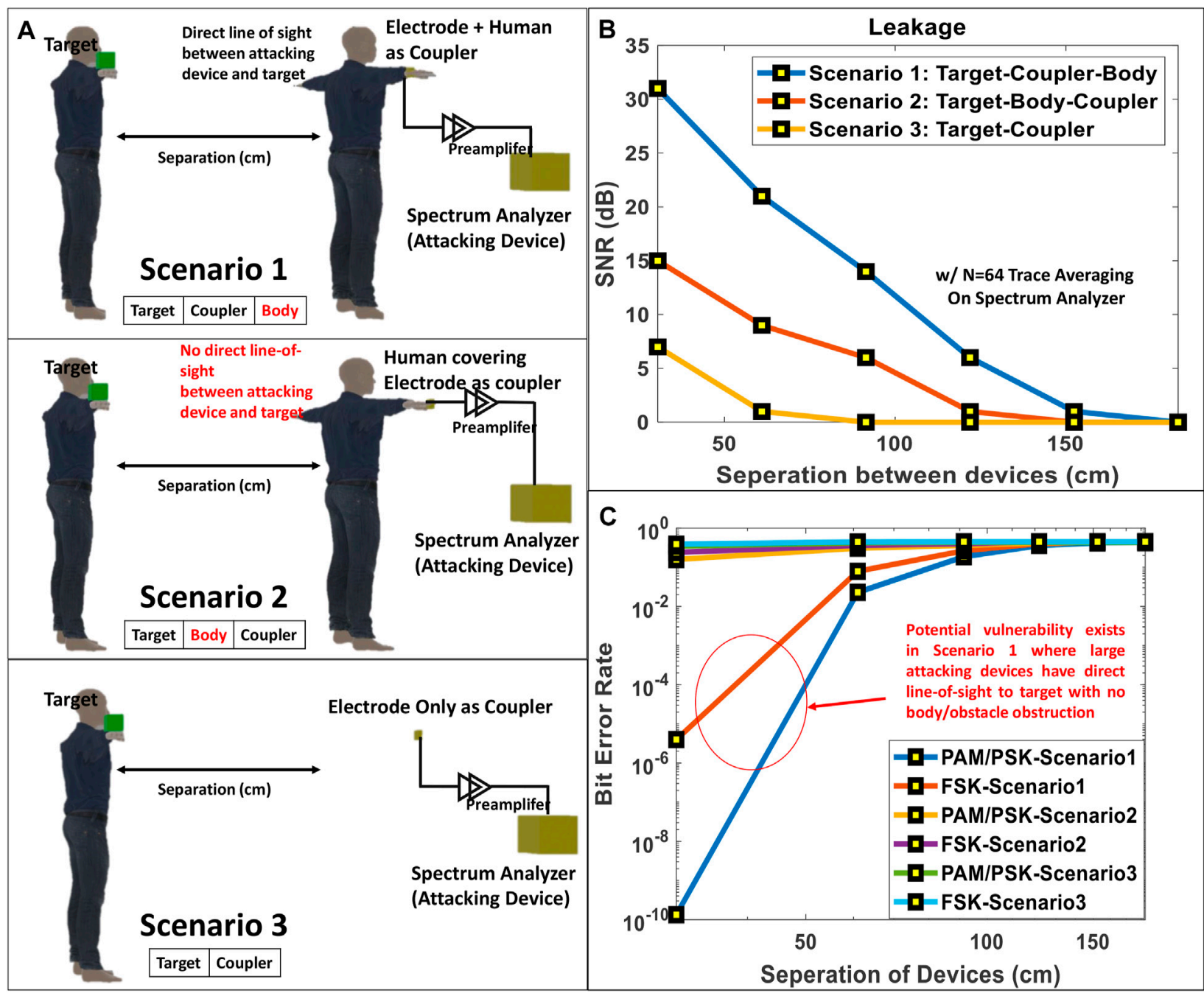

FIGURE 11 | (A) Measurement setup for leakage experiments - Scenario 1: leakage measured facing device on the body, Scenario 2: leakage measured facing the device (with the body in between two devices), and Scenario 3: leakage measured facing only the coupler (and no body). (B) SNR versus distance between the attacking device and a target device. (C) Theoretical bit error rate possible by attacking the device at a distance away.

HBC device transmits a test signal through their body. This signal represents the sensitive information that the user could potentially be protecting. The signal is received either using a stand-alone coupler or using the human body as an antenna.
Line-of-sight leakage describes a transmitter device with a direct coupling path to the measurement setup. Body obstructed constitutes the other scenario, where the device is on the opposite side of the body. In other words, the body-obstructed 


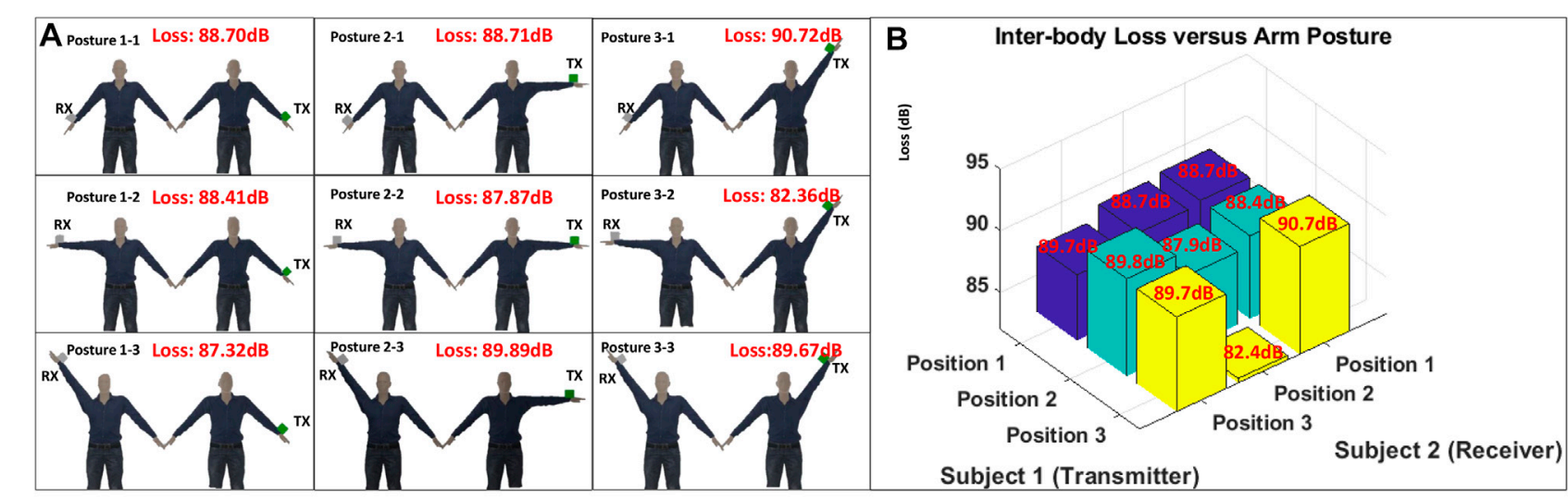

Experiment 1

FIGURE 12|Through-body interhuman experiment setup and postures characterized. (A) Experiment 1 includes the nine postures and loss values of interhuman channel loss. (B) Bar plot of averages of channel loss results.

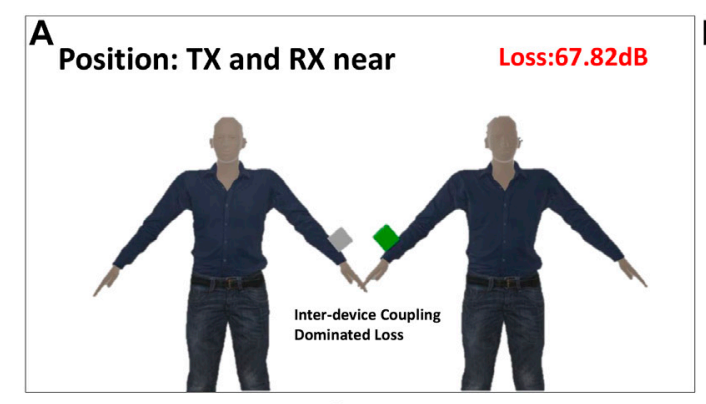

Experiment 2

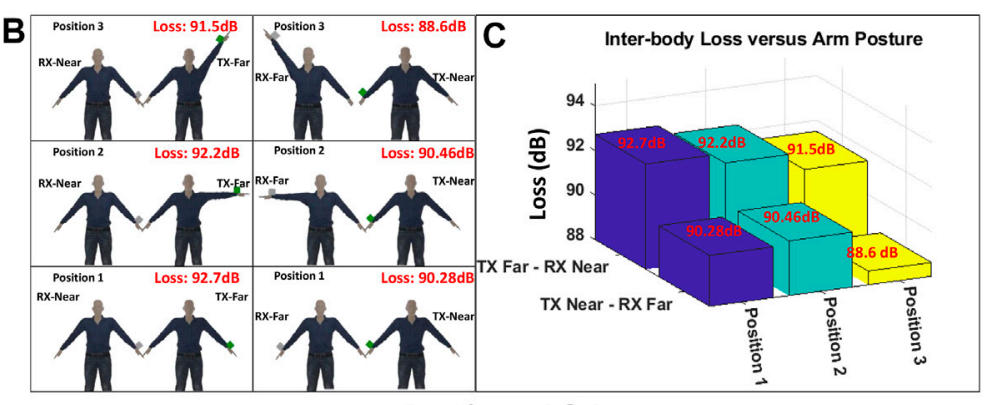

Experiment 3 \& 4

FIGURE 13 | Through-body interhuman experiment setup and postures characterized for (A) high inter-device coupling case where both users are wearing the device on the hand close to each other representing the best case channel, (B) postures of Experiments 3 and 4 and the corresponding channel postures where one user has the device close and the other on the opposite hand, (C) and a bar plot of results for Experiments 3 and 4.

measurements represent a device facing away from the attacker or a target with a transmitter that is either covered by the attacker's body or another object.

In the Scenario 1 experiment depicted by Figure 11A, the device is in direct line-of-sight contact with the attacking setup. This result is plotted in Figure 11B-Scenario 1, which shows the SNR vs. distance of the leakage signal that is available to the second subject. The second experiment is illustrated in Figure 11A Scenario 2 and has the identical setup as the first, with the exception of the body being in front of the coupler (and hence covering the coupler). The SNR for both experiments is measured relative to the noise floor of the receiver device, which is the spectrum analyzer (KT-N9000B-CXA Signal Analyzer) equipped with a high-impedance preamplifier buffer in this case.

From the results in Figure 11B, it can be seen that the leakage between the devices without the presence of a human is significantly lower than the case when the second person is used as the antenna to receive the signal. This shows that the signal leakage is significantly higher as the coupling between the two persons than the coupling between a person and the stand- alone coupler at the receiver end. Another important takeaway is that body-obstructed leakage has even lower SNR giving some directional protection from malicious attackers-suggests that the body covering can sometimes aids in security at a distance of $>30 \mathrm{~cm}$. Moreover, it is important to note that these measurements were performed using a spectrum analyzer with a $1 \mathrm{kHz}$ resolution bandwidth-which should be a good approximation for the fundamental limitation of what an attacker can detect. The noise floor of the spectrum analyzer is about $-139 \mathrm{dBm}$, which acts as the sensitivity limit at the receiver end-a trace averaging of $64 \mathrm{x}$ was used to derive the SNR results. From the signal-to-noise ratio, signal leakage per bit is used to calculate the theoretical BER (Figure 11C), should the attacker have a receiver with a sensitivity $-139 \mathrm{dBm} / \mathrm{Hz}$ (an idealistic receiver) at various distances away from the device they are attacking. This represents the number of bits that an attack can successfully recover as a function of distance away. This is the information theory-equivalent way of portraying the SNR data presented in Figure 11B. From Figure 11C, it can be observed that the communication is virtually impossible at 


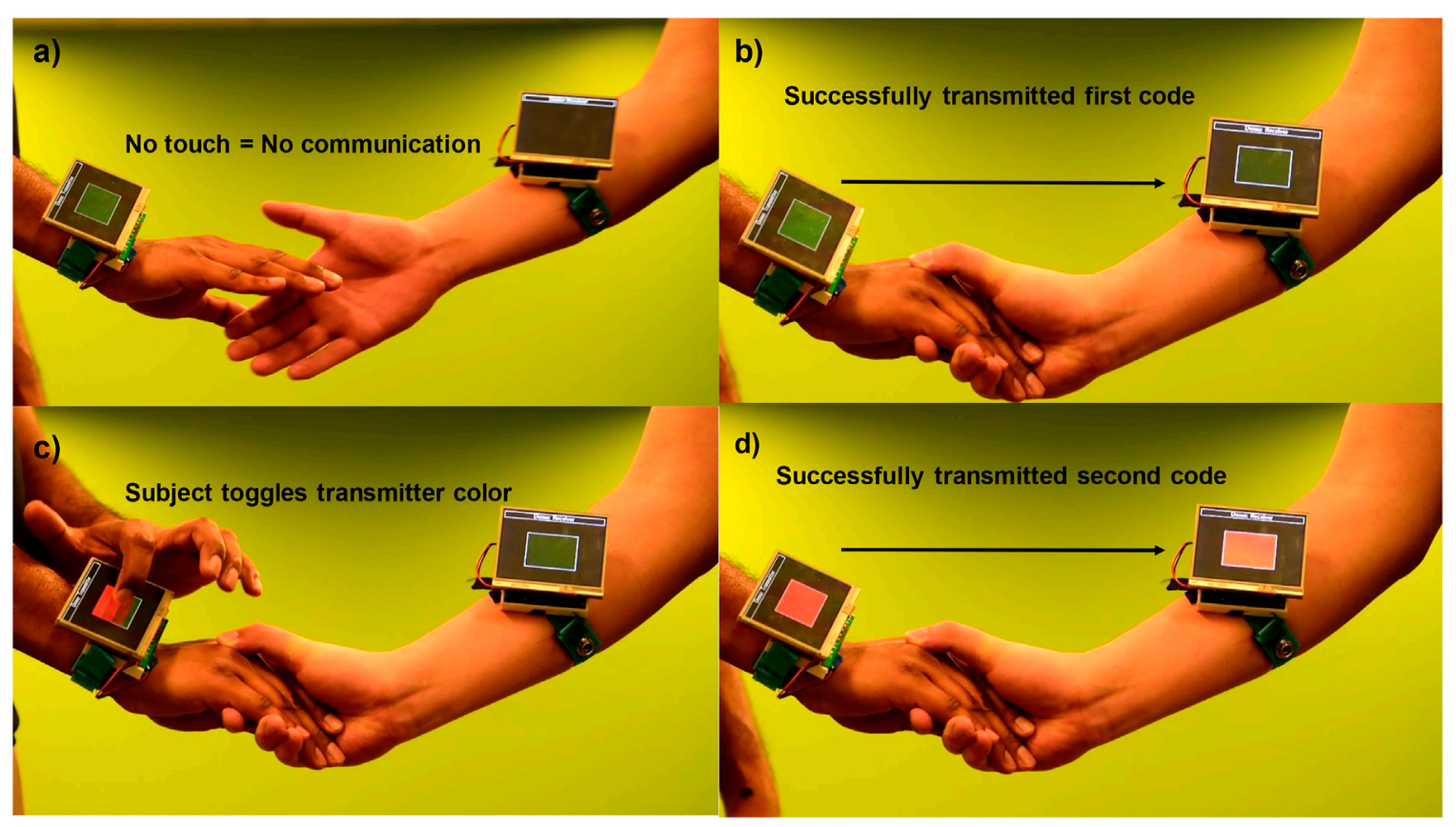

FIGURE 14 |Demonstration of inter-human information exchange through the BodyWire prototype. (A) No communication without physical touch illustrating signal confinement very close to the body. (B) Successfully transmitted initial color information from one to the other. (C) Subject toggles to different color information to transmit. (D) Second successful transmission of color information.

greater than $50 \mathrm{~cm}$ away in all scenarios and significantly lower for Scenario 2-where the transmitter is obstructed by another object or a body for extra security. These bit error rates are calculated for various digital modulations, namely PAM, PSK, and FSK of modulation order 2 and using the normalized SNR of the non-averaged spectrum analyzer results.

\subsection{Through-Body Interhuman Channel Loss Measurements}

This subsection details the results of through-body coupling channel characterization-that is the transfer of the signal between one human to another through direct contact. Although previous work such as Nath et al., (2020) has characterized the unintentional radiation at a distance of a meter, no other literature to the authors' knowledge has characterized the intentionally coupled body channel between two separate individuals (the through-body interhuman channel).

The results are presented for Experiment 1 in Figure 12 and Experiments 2, 3, and 4 in Figure 13. The results from all through-body interhuman channel loss show between 67 and $92.7 \mathrm{~dB}$ of channel loss depending on posture. The result from the second experiment is dominated by inter-device coupling and measured an average of $67 \mathrm{~dB}$ of channel loss with a range from 64-69 dB. These experiments show a typical increase in additional $15 \mathrm{~dB}$ of loss from the intra-body case across the board. This is expected as an additional coupling capacitance component that exists between the human contacts; variations coming from this coupling surface will directly reflect on the channel loss characteristics. It should be noted that the loss from Experiments 3 and 4 on average was a few $\mathrm{dB}$ more than that from Experiment 1 . This is can be explained by the theory developed in Datta et al., (2021) where body shadowing has a significant adverse effect on the signal level-interestingly enough, the channel actually improves for the configuration where the devices are further apart-as opposed to the scenario where one of the transmitters or receivers is sandwiched between the two humans.

\subsection{Wearable-Wearable HBC Demonstration}

Human body communication can enable many convenient new interactions between devices and humans. For example, secure authentication benefits greatly by being able to covertly transmit information through the body to a lock or secured device. Touchactivated communication systems would no longer require separate hardware or software overhead to incorporate a touch sensor, as the touch and communication are both integrated into the interaction. Information exchange between two wearable devices (smartwatches and smartbands) or a wearable device and a computer is an example of possible use cases of HBC. To show this technology in practice, we implemented a simple demonstration to illustrate the elegance and potential of BodyWire to benefit current human computer interaction applications. To the best of the authors' knowledge, this demonstration shows the smallest form factor and lowest frequency groundless state-of-the-art HBC system. 
One of the interesting use cases of $\mathrm{HBC}$ is through-body interhuman information exchange between two users during a touch event, such as a handshake. BodyWire shows a demo of two users using a groundless HBC system. The channel loss in an interhuman scenario is slightly higher than that in the intra-body case. The user with the transmitter device is able to toggle the color they want to send through the touch screen. The transmitter packetizes the 16-bit code corresponding to the color displayed on the screen and sends it through the human body using HBC. The receiver's signal conditioning front-end processes the noise and interferes for the digital processing unit. Next, the receiver decodes the compressed data in software to display the corresponding color on the display. The details of the demonstration are shown in Figure 14. Figure 14A shows that information is not transmitted between the devices when the persons are not in direct contact, showing signal confinement very close to the body. When the two persons perform a handshake, the green color information gets transmitted to the receiver as shown in Figure 14B. The transmitted color is changed (Figure 14C), and the subsequent color is received at the receiver end (Figure 14D).

\subsubsection{Hardware Implementation Details}

The HBC transceiver is fabricated on a custom two-layer printed circuit board with a size of $4.5 \times 5 \mathrm{~cm}$. The transceiver includes an analog front-end, which preconditions the signal by filtering and amplifying the signal for detection using a digital processing unit. The design techniques such as capacitive excitation and highimpedance capacitive termination at the receiver end described in previous sections are used in the design of the receiver. The digital processing of the signal from the analog front-end is performed using a TI TM4C123GH6PM microcontroller; BodyWire utilizes analog comparators in the microcontroller for interfacing with the analog hardware and detecting the bits. At the transmitter end, we use the same microcontroller to digitally synthesize the transmit signal and couple to the body directly.

\section{CONCLUSION}

In this article, we introduce BodyWire, which demonstrates communication between two wearable devices through electro-

\section{REFERENCES}

Author Anonymous (2021). Investigating Human Body Communications. Intel Newsroom.

Bae, J., Cho, H., Song, K., Lee, H., and Yoo, H.-J. (2012). The Signal Transmission Mechanism on the Surface of Human Body for Body Channel Communication. IEEE Trans. Microwave Theor. Techn. 60 (3), 582-593. doi:10.1109/tmtt.2011. 2178857

Bae, J., Song, K., Lee, H., Cho, H., and Yoo, H.-J. (2012). A 0.24-nJ/b Wireless Body-Area-Network Transceiver with Scalable Double-FSK Modulation. IEEE J. Solid-state Circuits 47 (1), 310-322. doi:10.1109/jssc.2011. 2170632 quasistatic human body communication. We carry out experiments to characterize the channel loss across different human subjects, environments, postures, and locations on the body. We discuss about the different factors such as ground isolation, device size, and packaging that can potentially affect the experimental results and provide suggestions about taking these factors into account while designing experiments to ensure repeatable measurements. The measurement results show that the channel loss variation across subjects, different postures, and environment is about $15 \mathrm{~dB}$, providing a design constraint for the receiver design. The results also show that the channel loss reduces by 3-4 dB in presence of a big object around the body, such as an office desk. This shows that the loss in an office/lab environment will be slightly better than that in an outdoor environment, which can be explained from the higher return path capacitance due to the parasitic coupling of the device ground with the environment. The almost constant channel loss for measurements across different locations of the body also shows the advantage of capacitive coupling over galvanic coupling. These characterizations were taken into account to design a prototype device demonstrating information exchange between two persons during a handshake, showing the smallest form factor wearable-wearable $\mathrm{HBC}$ information exchange according to the authors' best knowledge.

\section{DATA AVAILABILITY STATEMENT}

The raw data supporting the conclusion of this article will be made available by the authors, without undue reservation.

\section{ETHICS STATEMENT}

The studies involving human participants were reviewed and approved by the Purdue University IRB. The patients/ participants provided their written informed consent to participate in this study.

\section{AUTHOR CONTRIBUTIONS}

DY, SM, and SS contributed to the conception and design of the study, experiments, and writing. All authors contributed to manuscript revision and read and approved the submitted version.

Brain Bailey Microchip Technology Inc (2014). AN1391 Introduction to the BodyCom Technology. Microchip.

Buechley, L., Mellis, D., Perner-Wilson, H., Lovell, E., and Kaufmann, B. (2010). "Living Wall: Programmable Wallpaper for Interactive Spaces," in Proceedings of the 18th ACM International Conference on Multimedia, Firenze, Italy, October 25-29, 2010 (New York, NY, USA: Association for Computing Machinery), 1401-1402. MM '10.

Callejón, M. A., Naranjo-Hernández, D., Reina-Tosina, J., and Roa, L. M. (2013). A Comprehensive Study into Intrabody Communication Measurements. IEEE Trans. Instrumentation Meas. 62 (9), 2446-2455. doi:10.1109/TIM.2013. 2258766

Cho, N., Yoo, J., Song, S.-J., Lee, J., Jeon, S., and Yoo, H.-J. (2007). The Human Body Characteristics as a Signal Transmission Medium for Intrabody 
Communication. IEEE Trans. Microwave Theor. Techn. 55 (5), 1080-1086. doi:10.1109/tmtt.2007.895640

Cho, N., Yan, L., Bae, J., and Yoo, H.-J. (2009). A $60 \mathrm{~Kb} / \mathrm{s}-10 \mathrm{Mb} / \mathrm{s}$ Adaptive Frequency Hopping Transceiver for Interference-Resilient Body Channel Communication. IEEE J. Solid-State Circuits 44 (3), 708-717. doi:10.1109/ jssc.2008.2012328

Cho, H., Kim, H., Kim, M., Jang, J., Bae, J., and Yoo, H-J. (2015). "21.1 A 79pJ/b $80 \mathrm{Mb} / \mathrm{s}$ Full-Duplex Transceiver and a $42.5 \mu \mathrm{W} 100 \mathrm{~kb} / \mathrm{s}$ Super-regenerative Transceiver for Body Channel Communication," in 2015 IEEE International Solid-State Circuits Conference - (ISSCC) Digest of Technical Papers, San Francisco, CA, February 22-26, 2015, 1-3.

Das, D., Maity, S., Chatterjee, B., and Sen, S. (2019). Enabling Covert Body Area Network Using Electro-Quasistatic Human Body Communication. Sci. Rep. 9 (1), 4160. doi:10.1038/s41598-018-38303-x

Datta, A., Nath, M., Yang, D., and Sen, S. (2021). Advanced Biophysical Model to Capture Channel Variability for EQS Capacitive HBC. IEEE Trans. Biomed. Eng. 68, 3435-3446. doi:10.1109/TBME.2021.3074138

Dietz, P., and Leigh, D. (2001). "DiamondTouch: A Multi-User Touch Technology," in Proceedings of the 14th Annual ACM Symposium on User Interface Software and Technology, Orlando, FL, November 11-14, 2001 (New York, NY, USA: Association for Computing Machinery), 219-226. UIST '01.

Fukumoto, M., and Shinagawa, M. (2005). "CarpetLAN: A Novel Indoor Wireless(-like) Networking and Positioning System," in Proceedings of the 7th International Conference on Ubiquitous Computing, Tokyo, Japan, September 11-14, 2005 (Berlin, Heidelberg: Springer-Verlag), 1-18. UbiComp'05. doi:10.1007/11551201_1

Grosse-Puppendahl, T., Braun, A., Kamieth, F., and Kuijper, A. (2013). "SwissCheese Extended: An Object Recognition Method for Ubiquitous Interfaces Based on Capacitive Proximity Sensing," in Proceedings of the SIGCHI Conference on Human Factors in Computing Systems, Paris, France, April 27-May 2, 2013 (New York, NY, USA: Association for Computing Machinery), 1401-1410.

Habib, I., Berggren, N., Rehn, E., Josefsson, G., Kunz, A., and Fjeld, M. (2009). "DGTS: Integrated Typing and Pointing," in Human-Computer Interaction INTERACT 2009. Editors T Gross, J Gulliksen, P Kotzé, L Oestreicher, P Palanque, R Oliveira Prates, et al. (Berlin, Heidelberg: Springer Berlin Heidelberg), 232-235. doi:10.1007/978-3-642-03658-3_30

Hessar, M., Iyer, V., and Gollakota, S. (2016). "Enabling On-Body Transmissions with Commodity Devices," in Proceedings of the 2016 ACM International Joint Conference on Pervasive and Ubiquitous Computing, Heidelberg, Germany, September 12-16, 2016 (New York, NY, USA: Association for Computing Machinery), 1100-1111. UbiComp '16. doi:10.1145/2971648.2971682

Hwang, J.-H., Kang, T.-W., Kim, Y.-T., and Park, S.-O. (2016). Measurement of Transmission Properties of HBC Channel and its Impulse Response Model. IEEE Trans. Instrum. Meas. 65 (1), 177-188. doi:10.1109/tim.2015.2476236

Ikinamo (2009). Human Body as a Communication Medium. Chiba, Japan: DigInfo.

Kim, Y., Lee, S., Hwang, I., Ro, H., Lee, Y., Moon, M., et al. (2014). “High5: Promoting Interpersonal Hand-To-Hand Touch for Vibrant Workplace with Electrodermal Sensor Watches," in Proceedings of the 2014 ACM International Joint Conference on Pervasive and Ubiquitous Computing, Seattle, WA, September 13-17, 2014 (New York, NY, USA: Association for Computing Machinery), 15-19. UbiComp '14.

Lan, G., Ma, D., Xu, W., Hassan, M., and Hu, W. (2017). "CapSense: CapacitorBased Activity Sensing for Kinetic Energy Harvesting Powered Wearable Devices," in Proceedings of the 14th EAI International Conference on Mobile and Ubiquitous Systems: Computing, Networking and Services, MobiQuitous 2017, Melbourne, Australia, November 7-10, 2017 (New York, NY, USA: Association for Computing Machinery), 106-115.

Lee, C-H., and Selker, T. (2004). "iSphere: a Proximity-Based 3D Input Device," in ACM SIGGRAPH 2004 Posters, Los Angeles, CA, August 8-12, 2004 (New York, NY, USA: Association for Computing Machinery), 72. SIGGRAPH '04.

Lučev, Ž., Krois, I., and Cifrek, M. (2011). "A Capacitive Intrabody Communication Channel from $100 \mathrm{kHz}$ to $100 \mathrm{MHz}$," in 2011 IEEE International Instrumentation and Measurement Technology Conference, Hangzhou, China, May 10-12, 2011, 1-4. doi:10.1109/imtc.2011.5944311

Maity, S., Chatterjee, B., Chang, G., and Sen, S. (2018). "A 6.3pJ/b 30Mbps -30dB SIR-Tolerant Broadband Interference-Robust Human Body Communication
Transceiver Using Time Domain Signal-Interference Separation," in 2018 IEEE Custom Integrated Circuits Conference (CICC), San Diego, April 8-11, 2018, 1-4.

Maity, S., He, M., Nath, M., Das, D., Chatterjee, B., and Sen, S. (2019). Bio-Physical Modeling, Characterization, and Optimization of Electro-Quasistatic Human Body Communication. IEEE Trans. Biomed. Eng. 66 (6), 1791-1802. doi:10. 1109/tbme.2018.2879462

Maity, S., Yang, D., Redford, S. S., Das, D., Chatterjee, B., and Sen, S. (2020). BodyWire-HCI: Enabling New Interaction Modalities by Communicating Strictly during Touch Using Electro-Quasistatic Human Body Communication. ACM Trans. Computer-Human Interaction 27 (6), 1-25. doi:10.1145/3406238

Matsushita, N., Tajima, S., Ayatsuka, Y., and Rekimoto, J. (2000). "Wearable Key: Device for Personalizing Nearby Environment," in Digest of Papers. Fourth International Symposium on Wearable Computers, Atlanta, Georgia, October 18-21, 2000, 119-126.

Morgan, P., Singelée, D., Preneel, B., Marin, E., Garcia, F. D., Chothia, T., et al. (2021). Medtronic Conexus Radio Frequency Telemetry Protocol (Update C). CISA.

Nath, M., Maity, S., and Sen, S. (2020). Toward Understanding the Return Path Capacitance in Capacitive Human Body Communication. IEEE Trans. Circuits Syst.67 (10), 1879-1883. doi:10.1109/tcsii.2019.2953682

Park, J., and Mercier, P. P. (2015). "Magnetic Human Body Communication," in 2015 37th Annual International Conference of the IEEE Engineering in Medicine and Biology Society (EMBC), Milano, Italy, August 25-29, 2015 (ISSN), 18411558-18444615. doi:10.1109/embc.2015.7318739

Park, D. G., Kim, J. K., Sung, J. B., Hwang, J. H., Hyung, C. H., and Kang, S. W. (2006). "TAP: Touch-And-Play," in Proceedings of the SIGCHI Conference on Human Factors in Computing Systems (New York, NY, USA: Association for Computing Machinery), 677-680.

Park, J., Garudadri, H., and Mercier, P. P. (2017). Channel Modeling of Miniaturized Battery-Powered Capacitive Human Body Communication Systems. IEEE Trans. Biomed. Eng. 64 (2), 452-462. doi:10.1109/TBME. 2016.2560881

Rekimoto, J. (2002). "SmartSkin: An Infrastructure for Freehand Manipulation on Interactive Surfaces," in Proceedings of the SIGCHI Conference on Human Factors in Computing Systems, Minneapolis, MN, April 20-25, 2002 (New York, NY, USA: Association for Computing Machinery), 113-120. CHI '02.

Ruiz, J. A., and Shimamoto, S. (2005). "A Study on the Transmission Characteristics of the Human Body towards Broadband Intra-Body Communications," in Proceedings of the Ninth International Symposium on Consumer Electronics, 2005. (ISCE 2005), Macau SAR, June 14-16, 2005, 99-104.

Saadeh, W., Altaf, M. A. B., Alsuradi, H., and Yoo, J. (2017). A 1.1-mW Ground Effect-Resilient Body-Coupled Communication Transceiver with Pseudo OFDM for Head and Body Area Network. IEEE J. Solid-State Circuits 52 (10), 2690-2702. doi:10.1109/jssc.2017.2713522

Shin, S., and Lipton, J. (2018). Security Researchers Say They Can Hack Medtronic Pacemakers. Health \& Science.

Smith, J. R. (1996). Field Mice: Extracting Hand Geometry from Electric Field Measurements. IBM Syst. J. 35 (3.4), 587-608. doi:10.1147/sj.353.0587

Takahashi, M., Fernando, C. L., Kumon, Y., Takeda, S., Nii, H., Tokiwa, T., et al. (2011). "Earthlings Attack! a Ball Game Using Human Body Communication," in Proceedings of the 2nd Augmented Human International Conference, Tokyo, Japan, March 12-14, 2011 (New York, NY, USA: Association for Computing Machinery), 1-4. AH '11.

Valtonen, M., Maentausta, J., and Vanhala, J. (2009). "TileTrack: Capacitive Human Tracking Using Floor Tiles," in 2009 IEEE International Conference on Pervasive Computing and Communications, Galveston, TX, March 9-13, 2009, 1-10. doi:10.1109/percom.2009.4912749

Varga, V., Vakulya, G., Sample, A., and Gross, T. R. (2018). Enabling Interactive Infrastructure with Body Channel Communication. Proc. ACM Interact. Mob. Wearable Ubiquitous Technol. 1 (4), 1-29. doi:10.1145/3161180

Varga, V., Wyss, M., Vakulya, G., Sample, A., and Gross, T. R. (2018). “Designing Groundless Body Channel Communication Systems: Performance and Implications," in Proceedings of the 31st Annual ACM Symposium on User Interface Software and Technology, UIST '18, Berlin, Germany, October 14-17, 2018 (New York, NY, USA: Association for Computing Machinery), 683-695. 
Wegmueller, M. S., Oberle, M., Felber, N., Kuster, N., and Fichtner, W. (2010). Signal Transmission by Galvanic Coupling through the Human Body. IEEE Trans. Instrum. Meas. 59 (4), 963-969. doi:10.1109/tim.2009. 2031449

Williams, M. (2021). Panasonic's Wild New Technology Transmits Data by Human Touch \textbar PCWorld. PCWorld.

Yang, D., Mehrotra, P., Weigand, S., and Sen, S. (2021). In-the-Wild Interference Characterization and Modelling for Electro-Quasistatic-HBC with Miniaturized Wearables. IEEE Trans. Biomed. Eng. 68 (9), 2858-2869. doi:10.1109/tbme.2021.3082078

Zhang, Y., Zhou, J., Laput, G., and Harrison, C. (2016). "SkinTrack: Using the Body as an Electrical Waveguide for Continuous Finger Tracking on the Skin," in Proceedings of the 2016 CHI Conference on Human Factors in Computing Systems, San Jose, CA, May 7-12, 2016 (New York, NY, USA: Association for Computing Machinery), 1491-1503.

Zhou, J., Zhang, Y., Laput, G., and Harrison, C. (2016). “AuraSense: Enabling Expressive Around-Smartwatch Interactions with Electric Field Sensing," in Proceedings of the 29th Annual Symposium on User Interface Software and Technology, Tokyo, Japan, October 16-19, 2016 (New York, NY, USA: Association for Computing Machinery), 81-86. UIST '16.
Zimmerman, T. G. (1996). Personal Area Networks: Near-Field Intrabody Communication. IBM Syst. J. 35 (3.4), 609-617. doi:10.1147/sj.353.0609

Conflict of Interest: The authors declare that the research was conducted in the absence of any commercial or financial relationships that could be construed as a potential conflict of interest.

Publisher's Note: All claims expressed in this article are solely those of the authors and do not necessarily represent those of their affiliated organizations, or those of the publisher, the editors, and the reviewers. Any product that may be evaluated in this article, or claim that may be made by its manufacturer, is not guaranteed or endorsed by the publisher.

Copyright (C) 2022 Yang, Maity and Sen. This is an open-access article distributed under the terms of the Creative Commons Attribution License (CC BY). The use, distribution or reproduction in other forums is permitted, provided the original author(s) and the copyright owner(s) are credited and that the original publication in this journal is cited, in accordance with accepted academic practice. No use, distribution or reproduction is permitted which does not comply with these terms. 